\title{
Immunoassays of Methionine Adenosyltransferase Activity, S-adenosylmethione and Their Applications
}

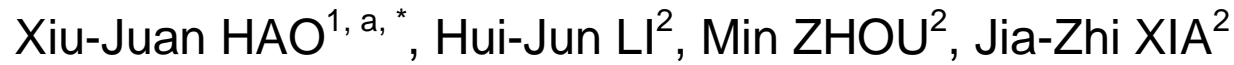 \\ ${ }^{1}$ Arthus Biosystems 2600 Hilltop Dr. Richmond, CA.94806, USA \\ ${ }^{2}$ Hunan SkyWorld Biotechnologies Co.Ltd 9 Panpan Rd. Changsha Economic and \\ Technological Development Zone, Changsha, Hunan 410100, China \\ aemail: sheryl.hao@arthusbio.com \\ ${ }^{*}$ Corresponding author
}

Keywords: S-adenosylmethionine, S-adenosylhomocysteine, Liver cancer, Methionine adenosyltransferase, Immunoassay.

\begin{abstract}
To understand how S-adenosylmethione (SAM) fluctuates in normal and cancerous liver cells, we developed novel methods for measuring methionine adenosyltransferase (MAT) activity and SAM. This method integrated MAT-catalyzed reaction and immunoassay that specifically and quantifies product SAM. IHC, IF and ELISA were used in measuring SAM and S-adenosylhomocysteine (SAH). SAM is mostly found in cytoplasm and less in nucleus whereas SAH is mostly found in nucleus. Levels of SAM and SAH were found to be reduced in cancer cells except for early stage when inflammation is boosted to fight against cancerous or other cell damages. $0.5 \mathrm{mM}$ methionine stimulated while S-nitrosoglutathione inhibited MAT-I/III activity and methylation index increased after methionine stimulationof L02 cells. Methionine inhibited while S-nitrosoglutathione had no effects on MAT-II activity and methylation index decreased after methionine stimulationof HepG2 cells. Immunoassays described provide better venues for further research on measuring MAT, SAM and SAH under various scenarios.
\end{abstract}

\section{Introduction}

S-adenosylmethionine(SAM, AdoMet) is an active form of methionine (Met) which is the key molecule in methionine cycle, the critical and basic metabolic pathway in all organisms. Most of the dietary methionine is converted into SAM in liver where up to $85 \%$ of all methylation reactions take place [1]. The first step in methionine metabolism is catalyzed bymethionine adenyltransferase (MAT, EC2.5.1.6)[2] also known as S-adenosylmethionine synthetase. Except for parasites that rely on host for living, cells from all organisms have MAT. MAT genes have been found to be exceptionally conserved throughout evolution. It was reported that there is $59 \%$ homology between human and E. coli MAT gene sequences. In mammals, three forms or isozymes of MAT have been identified that are encoded by three MAT genes. The MAT1a gene encodes $\alpha 1$ catalytic subunit. MAT-I is a tetramer of $\alpha 1$ subunits and MAT-III a dimer of the same subunits. Both MAT-I and MAT-III are present in adult liver cells. MAT-II is a heterotetramer formed by MAT2a encoding the catalytic subunit of $\alpha 2$ and MAT2b gene encoding $\beta$ subunit, present in cells other than liver, embryonic liver and hepatoma cells[3]. MAT isoforms differ in their kinetic and regulatory properties. MAT I and MAT-II have low $K_{\mathrm{m}}$ values for methionine, whereas MAT-III hasa high $K_{\mathrm{m}}$ for methionine [4]. MAT-I and MAT-III are reversibly inactivated by nitric oxide (NO) and hydroxyl radicals [5], whereas MAT-II is inhibited by physiological concentrations of SAM [6]. MAT-III is responsible for clearing 
methionine after a high-Met meal. MAT-I and MAT-II maintains the basal SAMlevelneeded by liver under fasting conditions [7].

MAT catalytic reaction in the body is divided into two steps: (1) Catalyze L-Metand adenosine triphosphate (ATP) to generate SAM and tripolyphosphate (PPPi). Both SAM and PPPi remain on the surface of MAT at this stage. (2)The phosphatase activity of MAT further decomposes PPPi to dimeric phosphoric acid (PPi) and inorganic monophosphate $(\mathrm{Pi})$.

Both ATP and SAM are considered two most common and important metabolic intermediates in vivo. SAM can only be synthesized by MAT. SAM is one of the few sulfur-containing active substances that carry extremely diverse and important biological functions in nature and is the key molecules in the methionine cycle. SAM is the sole methyl donor in the vital methylation process in human being and plays critical roles in transmethyl, transsulfuration, aminopropyl reactions, which mean SAM and MAT are directly involved in methylation-related cellular functions, polyamine synthesis, methythioadenosine regulated anti-inflammation, regulating the ratio of methionine and homocysteine. Therefore, they have significant impacts in various life essential metabolic reactions, cell proliferation, differentiation and apoptosis.

Current methods to measure MAT activity are: (1)high performance liquid (HPLC) and mass spectrometry (LC/MS/MS) method to determine of SAM[3,4] synthesized; (2)malachite green and ammonium molybdateto determine the content of inorganic phosphorus $\mathrm{Pi}[10]$. Method (1) is a direct method yet it has a few limitations: (a) Inaccurate as synthesized SAM cannot be measure immediately after it was synthesized due to the lengthy and harsh sample preparation, manipulation and measurement processes, the highly instable nature of SAM makes (1) more undesirable. (b) Newly synthesized SAM is associated with MAT and not completely released till later; chromatography technology may exclude this portion of SAM. (c) Lack of specificity especially HPLC where distinguishes SAM and peaks of other metabolites such as SAH can be a challenging task. Method 2 hasthe following limitations: (a)PPPi, PPi and Pi may coexist; malachite green with ammonium molybdatecan also bind to PPPi and PPi leading to inaccurate results. (b) Sensitivity is low. (c) MAT triphosphatase activity needs a configuration change, so the reaction product of triphosphatase $\mathrm{Pi}$ undergoes delay effect, plus it is an indirect method, thus high variability exists. (d) MAT triphosphatase activity is greatly influenced by Met, which made it less reliable. (e) Using MAT's phosphatase activity to reflect its SAM synthetic ability, which poses additional problems since it is very likely MAT's phosphatase and SAM synthesis activities are not correlated and these two activities are regulated differently. Thus it is not always a good idea to use MAT hydrolysis phosphatase activity to reflect MAT SAM synthesis activity.Both methods are inaccurate and cannot sensitively reflect subtle changes of MAT activity under manycircumstances. Therefore, a fast, convenient, accurate, specific method to measure MAT activity is in great demand. In this paper we describe a novel and much better way to measure MAT activity.

Our interest has been focused on investigation of how MAT activity is related to methylation index (Ratio of SAM to SAH) in liver cells. A more accurate and sensitive way of measuring MAT activity is essential for us to understand how MAT genes are regulated and how reserve of SAM in cells changes, especially in liver which is the key organ of methionine cycle and SAM metabolism. With the availability of two liver cell lines that express different types of MAT genes and immunoassay we developed, measuring MAT activity, SAM and SAH become possible and help address some important processes of methionine cycle in the normal and cancerous hepatocytes. In the current work, immunofluorescence, immunohistochemistry and ELISA for SAM 
and SAH metabolites from liver cell lines and tissues were performed under different scenarios hoping to learn more about MAT activity and methylation index in liver.

\section{Materials}

L-Met,SAH, adenosine (Ade), MTA, ADP, ATP, S-nitrosoglutathione (GSNO), Diaminobenzidine (DAB),trypsin are from Sigma. MTA is from ABXBIO, Beijing. Fetal bovine serum, RPMI 1640 medium, DAPI(4',6-diamidino-2-phenylindole) are from Life technologies. SAM ELISA kit, PLL-aza-SAM, monoclonal antibodies against SAM and SAH, HRP-conjugated anti-SAM and anti-SAH antibodies Aza-SAM, SAM-Na, are from Arthus Biosystems. Tetramethylbenzidine(TMB) is from Yingchuang, Huzhou. FITC-labelled goat anti-mouse IgG is from abcam.Tissue chips (multi-organ tumor, its normal adjacent tissues (NAT) and normal tissues BCN963a, BN242b, TP242b) from Alenabio.

\section{Methods}

\section{Principle of the MAT Assay}

MAT catalyzes L-Met and ATP to generate SAM and PPPi. MAT has delayed triphosphatase activity after SAM synthesis. Therefore, PPPi is then further broken down into pyrophosphate and phosphoric acid. Its activity is dependent on $\mathrm{Mg}^{2+}$ and $\mathrm{K}^{+}$, enzymatic reaction can be shown as follows:

$$
\text { ATP + L-Met---(MAT, } \left.\mathrm{Mg}^{2+}, \mathrm{K}^{+}\right)--->\mathrm{SAM}+\mathrm{PPi}+\mathrm{Pi}
$$

The assay is designed to calculate MAT activity through measuring the concentration of the main product SAM. The chemical reaction above and the competition enzyme-linked immune-absorbent assay (cELISA) are carried out simultaneously, so that the SAM synthesized from the chemical reaction competes with antigens coated on the micro-titer plate for the HRP-conjugated anti-SAM antibody the minute it is generated. SAM is quantified by and proportional to the amount of the HRP-anti-SAM antibodies that were made not to bind to the micro-titer plate.

\section{Specificity of Anti-SAM Monoclonal Antibody}

cELISA was used to allow analogs such as SAH, Ade, MTA, ADP, ATP, L-Metto be used to compete with immobilized SAM antigen in binding HRP-conjugated anti-SAM antibody.

\section{Procedure of MAT Assay}

Add MAT samples, extra amounts $(>0.5 \mathrm{mM})$ of ATP and Met substrates prepared in a buffer containing $150 \mathrm{mM} \mathrm{KCl,20mM} \mathrm{MgSO} 4$ and $100 \mathrm{mM}$ Tris $\mathrm{pH} 7.42,1: 30,000$ HRP-anti-SAM to $0.05 \mu \mathrm{g} / \mathrm{ml}$ PLL-aza-SAM coated microtiter plate. Incubate it at $37^{\circ} \mathrm{C}$ for 60 minutes before adding TMB substrates for 15 minutes. Stop TMB color development and measure OD450 with (Thermo Scientific MULTISKAN FC). Definition of MAT activity: The amount of MAT required to produce $1 \mathrm{nM}$ at $37^{\circ} \mathrm{C}$ per minute is defined as a unit of enzyme activity.

\section{Immunohistochemistry (IHC) Procedure}

To stain cells or tissue sections, slides are blocked with blocking buffer for 30 minutes at room temperature and incubated with 1:50 -1:400 diluted antibodies. After rinsing with TBST, slide was incubated with HRP labeled goat-anti-mouse IgG for 2 hours. 
After washed with TBST, slides were treated with DAB reagents to visualize staining. Slides can then be counterstained with hematoxylin (HE) lightly, dehydrated, and mounted for microscopy examination and photography.

\section{MAT Activities of L02 and HepG2 Cells in Response to GSNO and Met}

Cells were cultured in 10\% FBS RPMI 1640 medium. When about $80 \%$ confluence was achieved, the cells were washed with PBS. Serum-free MEM medium containing $2 \mathrm{mM}$ GSNO was added and incubated for 30 minutes. Removed medium and washed again. Added different amount of Met in MEM medium containing 5\% FBS and 2mM glutamine and placed in $37^{\circ} \mathrm{C}$ incubator with $5 \% \mathrm{CO}_{2}$ and proper humidity for $24 \mathrm{~h}$. Cells were digested from the flasks with $0.25 \%$ trypsin solution, then washed with PBS and counted viable cells after Trypan blue staining. Quantification of intracellular SAM was immediately performed as follows: $1 \mathrm{ml}$ cell suspensions from the above were sonicated in ice bath for 5 minutes to break down cell membrane and then centrifuged at $15000 \mathrm{~g}$ at $4^{\circ} \mathrm{C}$ for $15 \mathrm{~min}$, supernatant is used to measure SAM using cELISA with about 10ul sample. The results were normalized as $2 \times 10^{7}$ cells.

\section{Immunofluorescence (IF) Cell Smear}

L02 and HeptG2 cells were treated with Met as described above after cells were properly cultured on glass slides. Cells were fixed with $80 \%$ ice acetone for 20 minutes followed by washing with PBS for 3 times. Slides were blocked with $0.5 \%$ skimmed milk solution. 50 $\mu \mathrm{l}$ of 1:400 diluted monoclonal anti-SAM antibody 118-6 and anti-SAH antibody $301-1$ were added at $37^{\circ}$ for $1 \mathrm{~h}$ and then washed for 3 times. 1:500 diluted FITC-labelled goat anti-mouse IgG were added and incubated at $37^{\circ}$ for $45 \mathrm{~min}$ in dark and then washed for 3 times. To counter stain nuclei, slides were incubated with $100 \mu 10.5 \mathrm{mg} / \mathrm{ml}$ DAPI for $20 \mathrm{~min}$ and washed 5 time before photographing under confocal microscope.

\section{Results}

\section{Monoclonal Antibody against SAM is Highly Specific}

Results from competitiveELISA usingmonoclonal antibody against SAM 84-3 indicate the cross reactions with analogs L-Met, SAH, Ade, ADP, ATP and MTA are all $<1 \%$ ( Fig. 1). It is very important to ensure that the specificity of the anti-SAM antibody is super before employing the immunoassay to monitor SAM synthesis reaction as the substrates ATP and L-Met and the SAM synthesized by MAT will be in the same system with the 84-3 or 118-6 antibody is competitively bound. Any cross reaction of Met or ATP with anti-SAM antibody will cause the inaccurate competition of SAM produced to bind HRP-anti-SAM antibody. Therefore, highly specific antibody will definitely make the measurement of MAT activity described in this investigation much accurate and reliable. On the other hand, highly specific antibody is especially critical wile planning to use it in immunohistochemistry (IHC) as there are a myriad of bio-molecules in cells and the background staining could be high if specificity is not ideal. With the level of specificity of the anti-SAM antibodies, using these antibodies in IHC, ELISA and MAT enzymatic activity assay will help elucidate many important questions with no doubt.

\section{Novel Method for MAT Activity Assay}

As shown inFig. 2, dose-dependent competition was observed as a sample was added to the cELISA using HRP-anti-SAM antibody. Any SAM from a sample competes with 
the coated SAM hapten to bind HRP-conjugated anti-SAM antibody. The sample is the product of the following reaction: MAT was added to Met and ATP in a proper buffer at $37^{\circ} \mathrm{C}$. It indicates that anti-SAM specifically binds to physiologically produced SAM or SAM synthesized by MAT. SAM measured is sensitive to the change of MAT activity. The SAM synthesized by MAT in the reaction is proportionately related to reaction time within 60 minutes. After 60 minutes, the reaction plateau was reached under the circumstances investigated. We have also found a way to boost MAT activity detection sensitivity. If the MAT activity ofa sample is low or hard to detect, let the sample and the MAT substrates react first at $37^{\circ} \mathrm{C}$ for $20 \mathrm{~min}$. Then add HRP-antibody followed by incubating the plate at $37^{\circ} \mathrm{C}$ for about $40 \mathrm{~min}$. This two-step method is useful when MAT activity from a sample is relatively low. If there is no such a concern, MAT enzymatic reaction and cELISA can be carried out at the same time, i.e. one-step reaction. The MAT activity assay described here has several advantages: (a) It can most accurately and sensitively reflect dynamic aspect of MAT activity; (b) No method is better than this one-step method to promptly and accurately measure SAM synthesized due to the extremely unstable nature of SAM; (c) The fastest way to measure MAT activity. This novel, accurate, convenient and fast way to measure MAT activity allows any laboratory to be able to do it easily and quickly without needing any special and costly equipment yet the results are the more reliable than those from measuring $\mathrm{Pi}[10]$ or SAM by HPLC or MS[8,9].

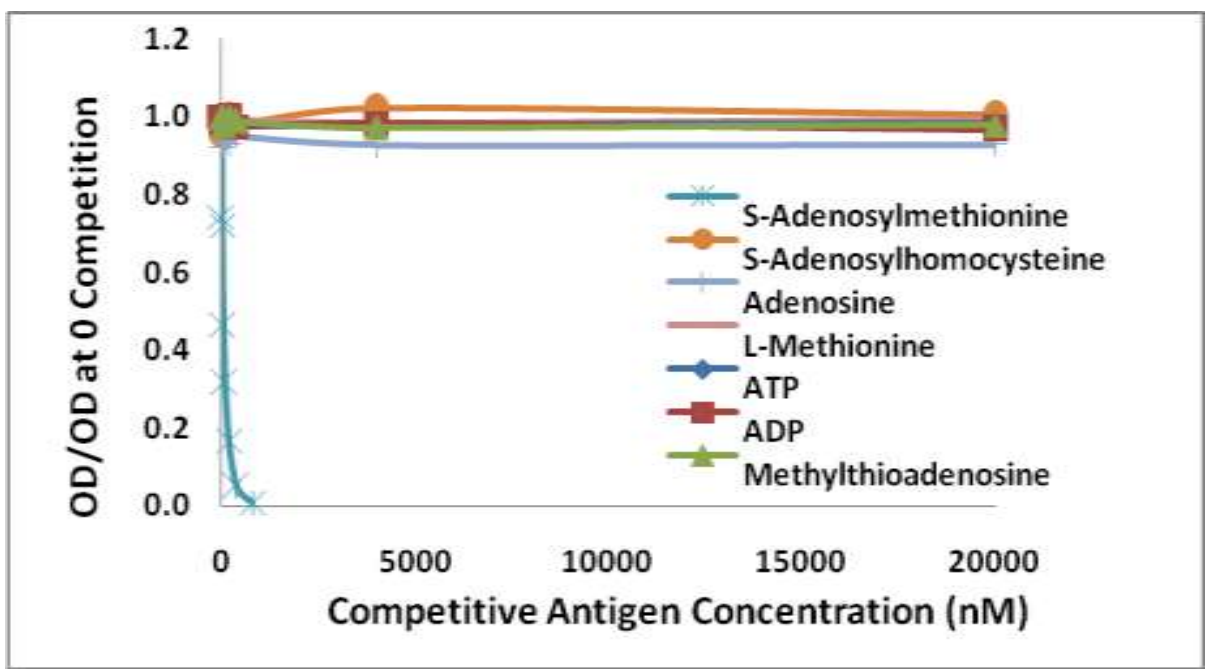

Figure 1. Competitive ELISA using anti-SAM monoclonal antibody $118-6$ the $0.1 \mu \mathrm{g} / \mathrm{ml}$ of SAM coating standard was coated into 96 wells. Serial dilution of SAM standard, SAH, Ade, L-Met, MTA, ADP, ATP and 1:35000 diluted 118-6 were added. HRP conjugated goatanti-mouse IgG antibody was used to develop the color before measuring OD450. 


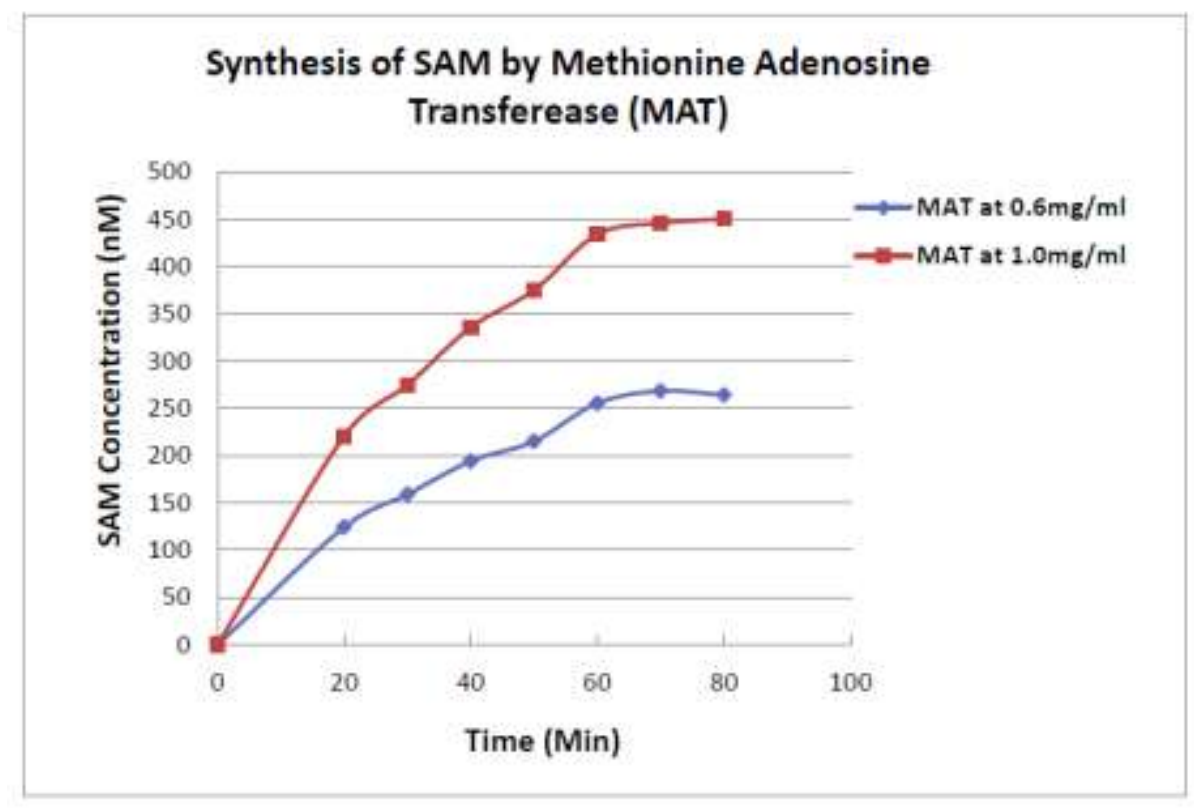

Figure 2. Different amounts of MAT were tested for catalyzing Met and ATP to synthesize SAM in a buffer incubated at $37^{\circ} \mathrm{C}$ for $20-80$ minutes.

\section{Localization of SAM and SAH and Changes of Their Expressions in Cancers}

In order to find out where in cells SAM and SAH are mostly located, different amount of antibodies were used and different levels of HE staining (blue-stained nuclei) were tested with normal and cancer tissue slides.Positive staining was shown in brown areas.Fig. 3A showed mostly cytoplasmic and less nuclear SAM specific staining in normal liver cells. Fig. 3B showed mostly nuclear and less cytoplasmic SAH in normal liver cells.HE counter-stain (light blue) was performed briefly to avoid interfering nuclear staining. As SAM and SAH are small metabolites that exist with great dynamics, results may vary with the methods and time tissue slides were fixed and prepared. Different cancer types, particular cases and samples may also give different results because each case is unique in terms of stages and types, patient overall health conditions, treatments and complications of other diseases and so on, which may contribute to various results. Therefore, more and better controlled sample preparation and studies are important and needed. We performed IHC staining with 3 different tissue chips with 16 different tissues and organs. Our findings indicated that in most cancers SAM and SAH levels were reduced except for a few case when SAM staining in early stage of lung cancer (Fig.3D1, 3D2 56-year old man with squamous cell carcinoma, TNM classification T2N0M0, stage I) was stronger than normal lung tissue. In lungadenocarcinoma (T2N0M0, II), SAM was not increased compared to its control (Fig.3E1, 3E2). In the case of liver, cerebrum, kidney, esophagus, etc, introcellular SAM was reduced in cancer cells 

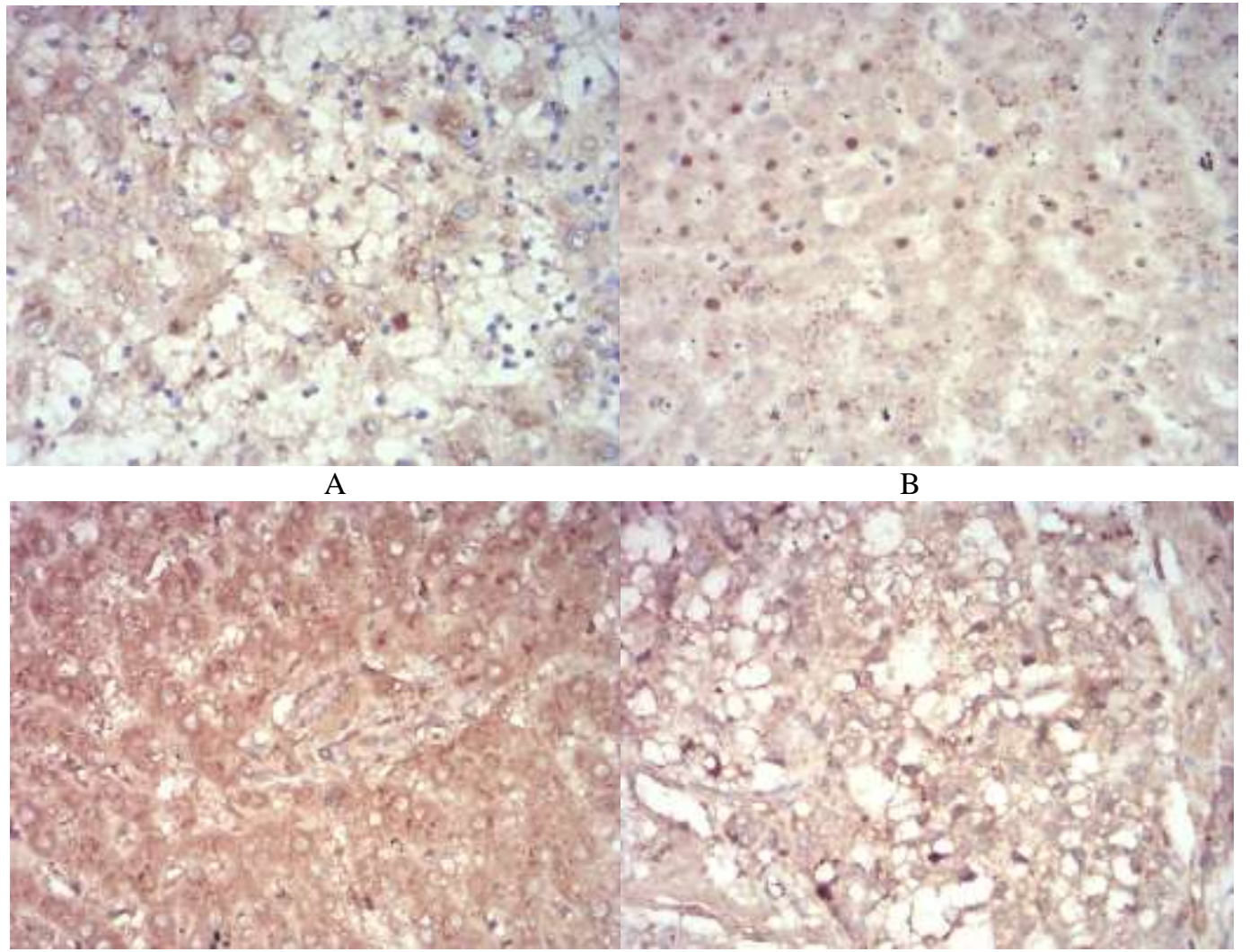

C1

$\mathrm{C} 2$
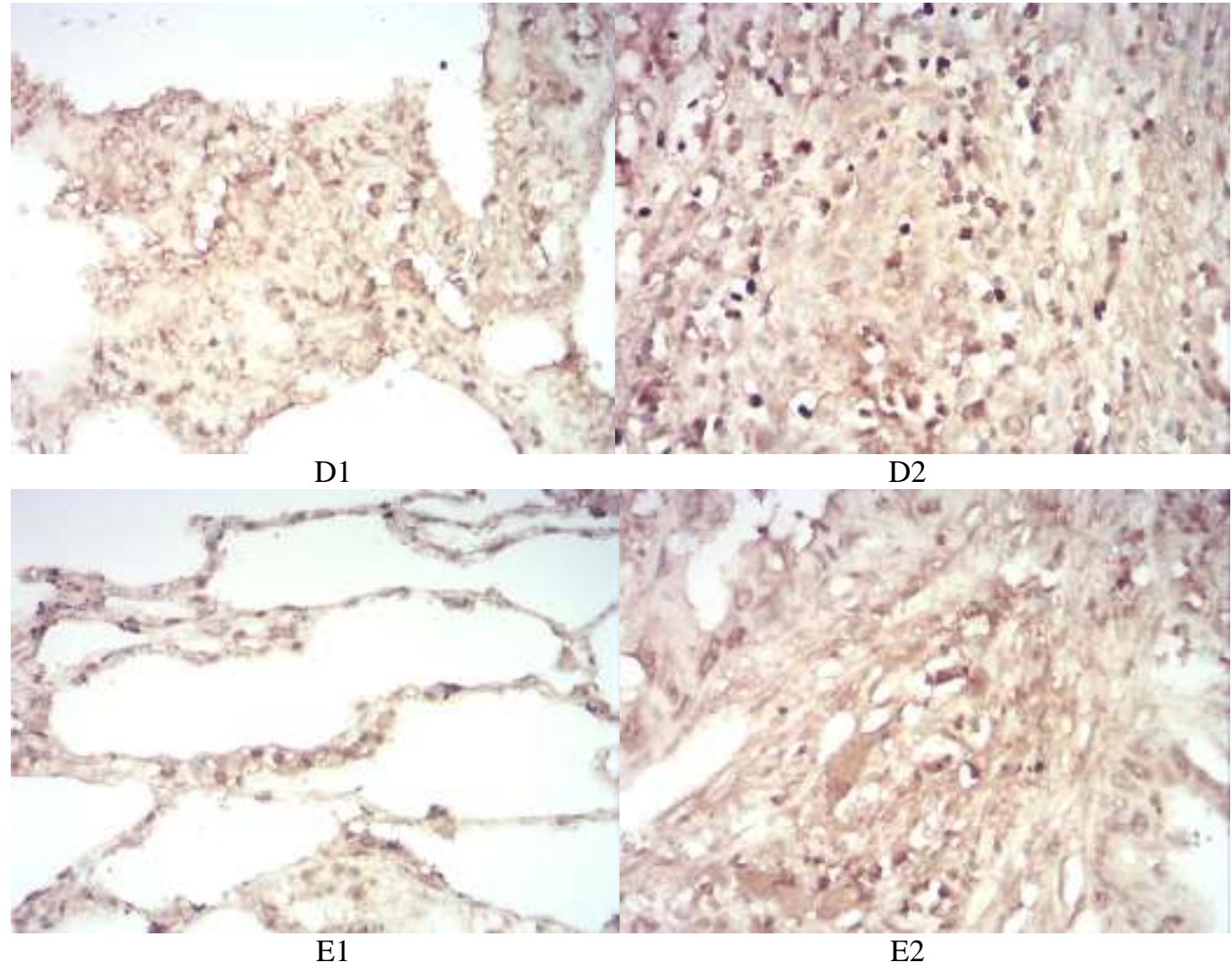


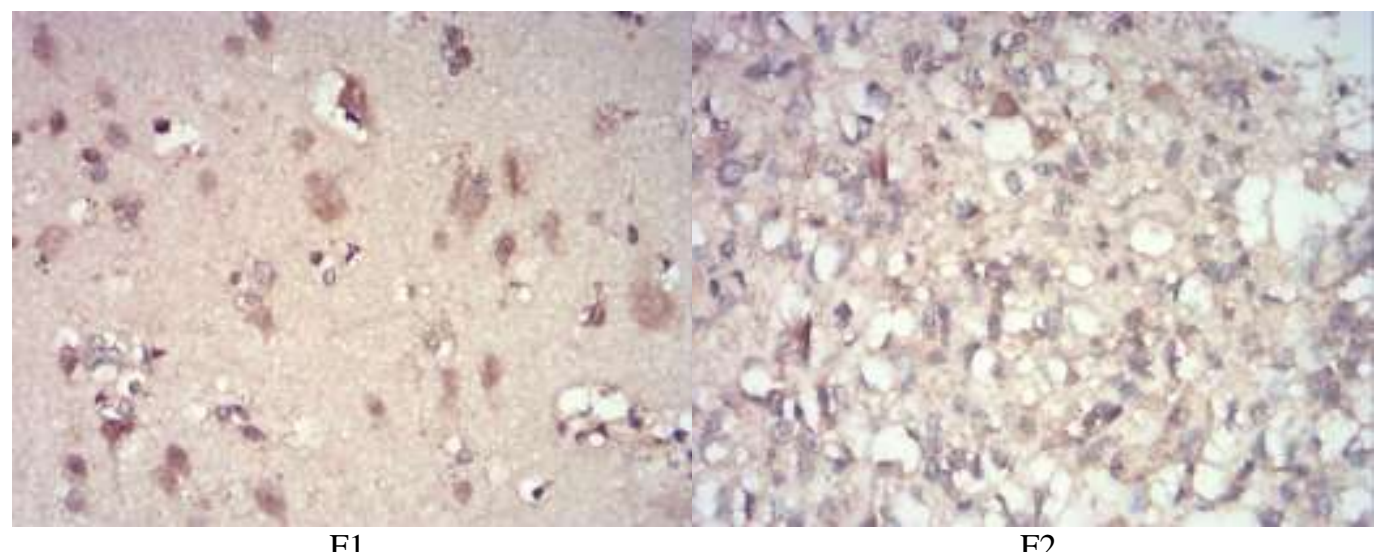

F1

Figure 3. Results of immunohistochemistry in paraffin-embedded tissue sections and chips. Use of anti-SAM antibody 118-6 (A) and anti-SAH antibody 301-1 (B) diluted at 1:200 with normal liver tissues. C1-F2 show results from tissue chips stained with 118-6. C1: NAT of C2; C2: Hepatocellular carcinoma (T2N0M0, II) of M39(male, 39 years old); D1: NAT of D2; D2: Lung squamous cell carcinoma (T2NOM0, I) of M56;E1: NAT of E2; E2:Lungadenocarcinoma (T2N0M0, II) of M39;F1: NAT of F2; F2: Cerebrum astrocytoma of M18. All pictures are taken under 400magnification.

\section{Regulation of MAT Activity by Met and GSNO}

Intracellular SAM level measured by ELISA was used to reflect MAT activity. Fig.4 shows the changes of MAT activities when Met and GSNO were tested on L02 and HepG2. After $0.5 \mathrm{mM}$ Met stimulation for 24h, SAM synthesis in L02 cells increased whereas $2 \mathrm{mM}$ Met did not stimulatebut inhibited MAT activity. GSNO reversed Met's stimulating effects in L02. Met inhibited MAT-II activity in HepG2 cells and GSNO did not seem to have any effects on HepG2 cells. The level of SAM was lower in HepG2 than in L02, if confocal microscopy (Fig. 5) confirmed these results.

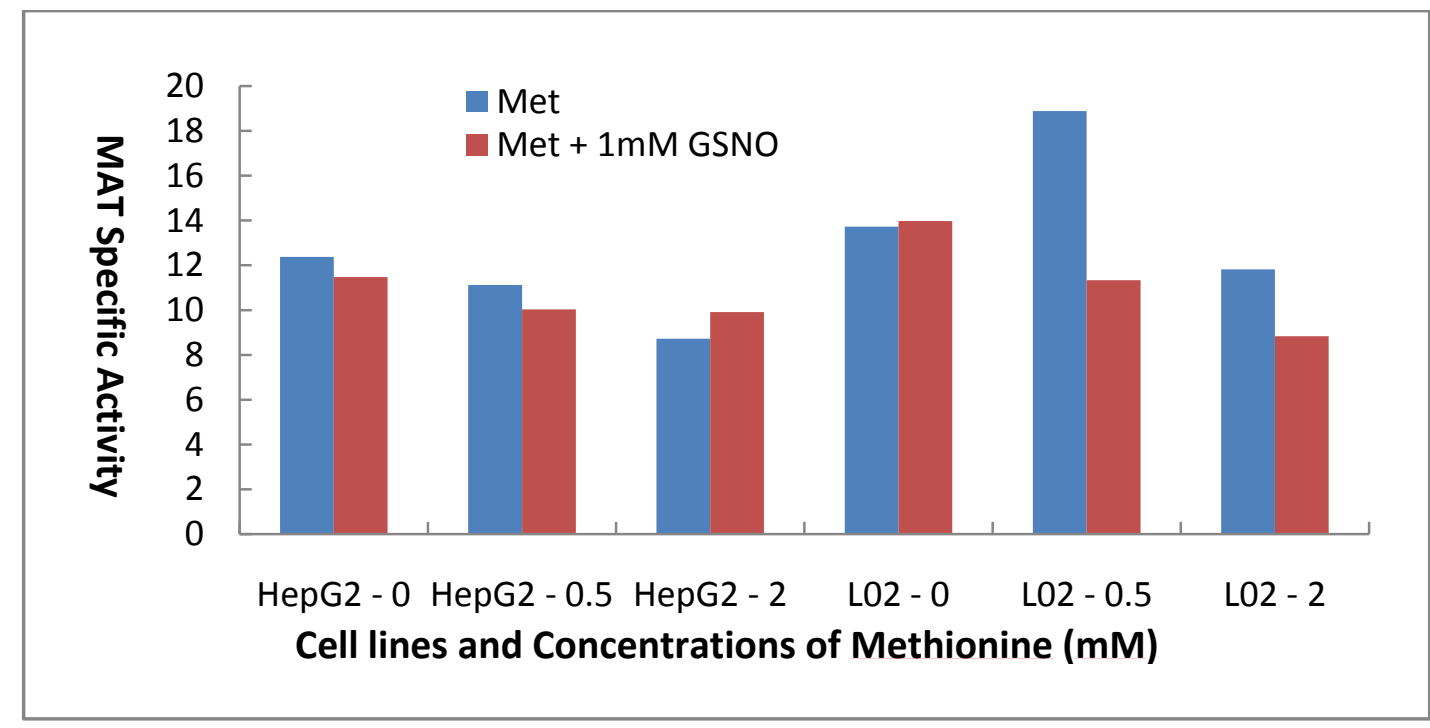

Figure 4. Regulation of MAT activities by Met and GSNO in L02 and HepG2 cellsConcentrations of Met and GSNO used are indicated. The number of cells from all groups was normalized to be $2 \times 10^{7}$. MAT specific activity was defined as the amount of SAM synthesized in nM per minute per $2 \times 10^{7}$ per procedure described. The results showed the average of two separate experiments.

It is believed that Met regulates enzymatic activity of MAT, which is critical in regulating the level of SAM and plays critical roles in Met cycle and epigenetic research. Therefore, specific antibodies against SAM and SAH were used in IF before and after Met stimulation of $\mathrm{L} 02$ and HeptG2 cells to see how cells react to Met 
stimulation and whether methylation index changes as a result. Fig.5A-5D showed results of changes of SAM expression and 5E-5H showed changes of SAH expression. After $0.5 \mathrm{mM}$ Met stimulation of L02 cells for about $24 \mathrm{~h}$, SAM showed more prevalently expressed in cytoplasm and slightly seen in nuclei (Fig. 5B). In the case of HepG2 cells, SAM expression was obviously inhibited by Met stimulation (Fig.5D). The changes of SAM level are closely related to the regulation of MAT activity by Met. In hepatocellular carcinoma cells HepG2, SAH expression was trivial and unnoticeable compared to that in normal liver cells L02 (Fig. 5E), indicating defective methylation or almost missing methylation process in the HepG2 cells, which likely contributes to HepG2 carcinogensis or its dedifferentiation. With $0.5 \mathrm{mM}$ Met stimulation of L02 for 24h, SAH expression was reduced while SAM was increased indicating methylation index was increased (Fig. 5A,5B,5E,5F). After 0.5mM Met stimulation of HeptG2 for $24 \mathrm{~h}, \mathrm{SAH}$ expression was significantly increased in nuclei and the methylation index was obviously reduced as SAM was reduced (Fig. 5C, 5D, 5G, 5H). Compared to HepG2 cells prior to stimulation, Met stimulation seemed play a big role in turning on its methylation process, probably by activate DNA methytransferases as the burst of SAH occurred in nucleus.

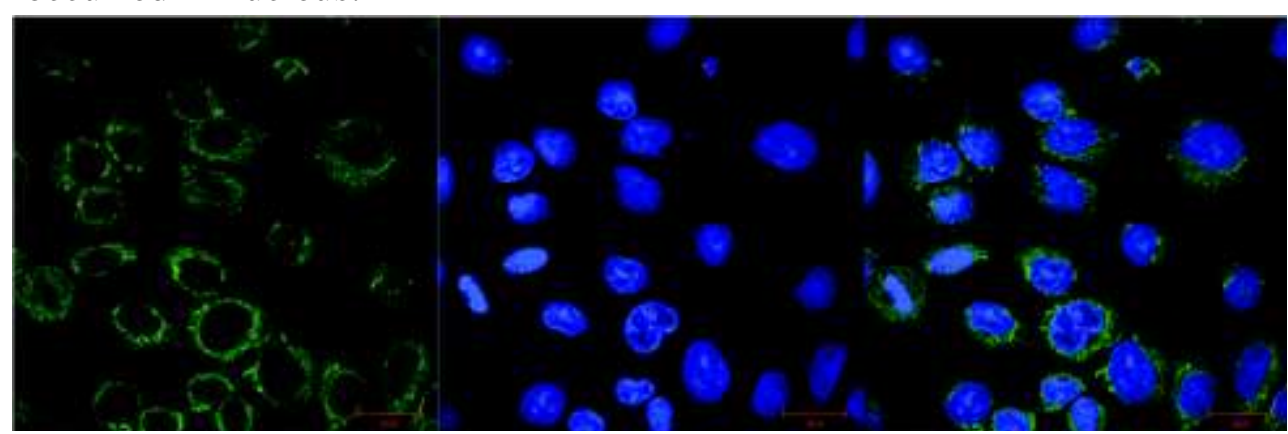

A

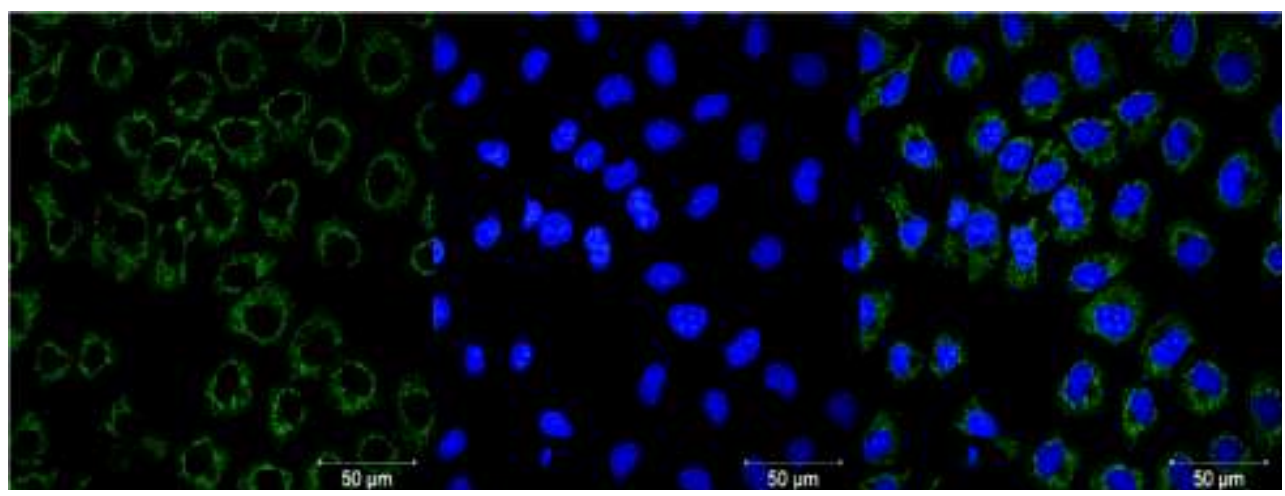

$\mathrm{B}$

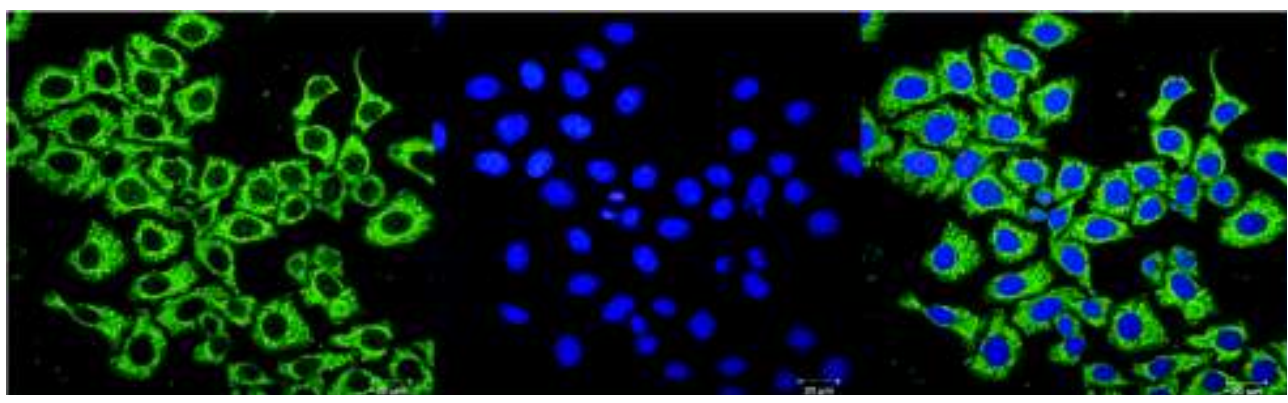

$\mathrm{C}$ 


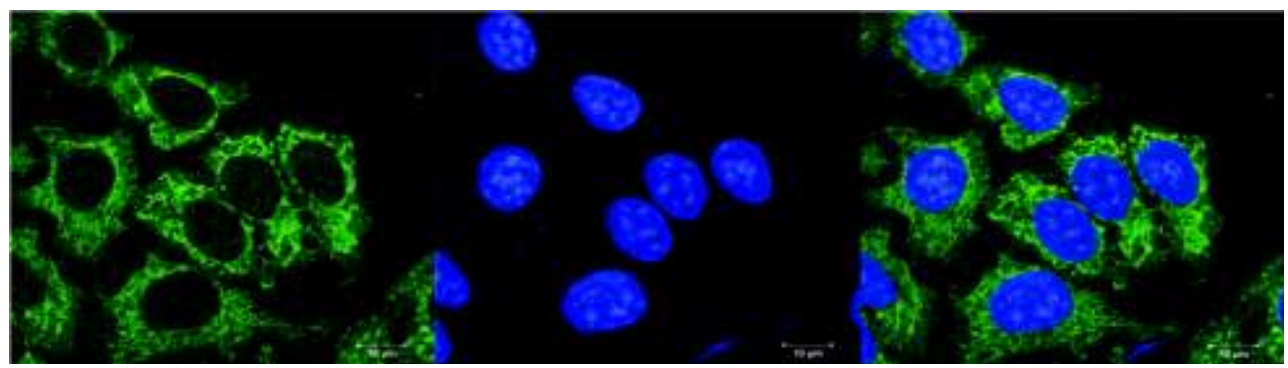

$\mathrm{D}$

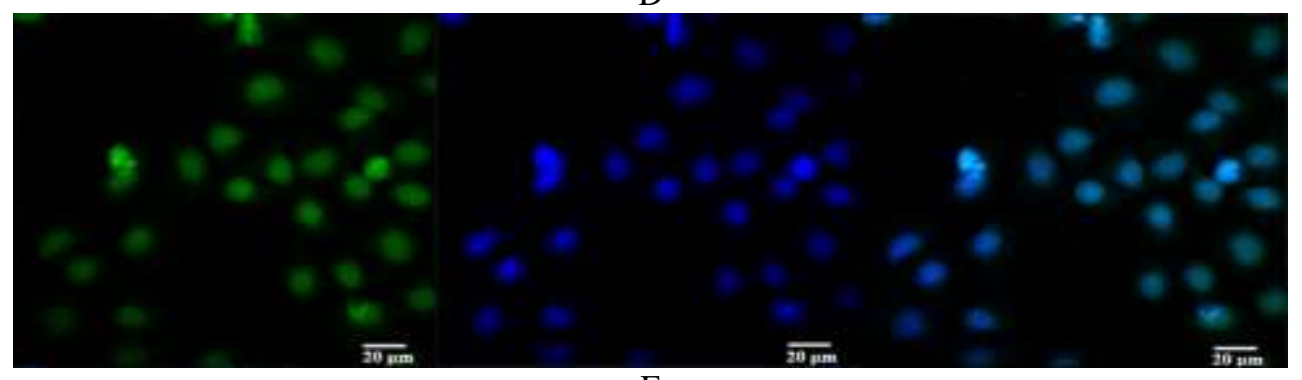

$\mathrm{E}$

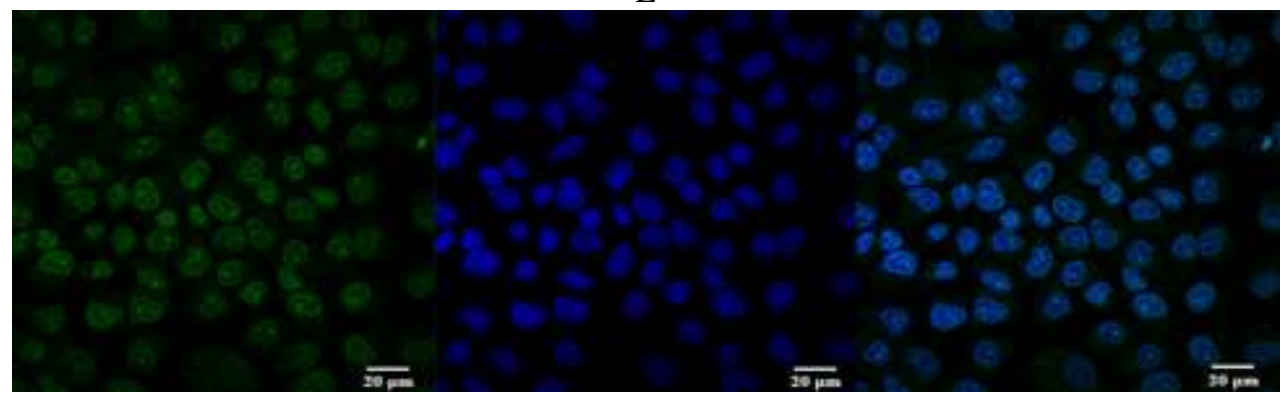

$\mathrm{F}$

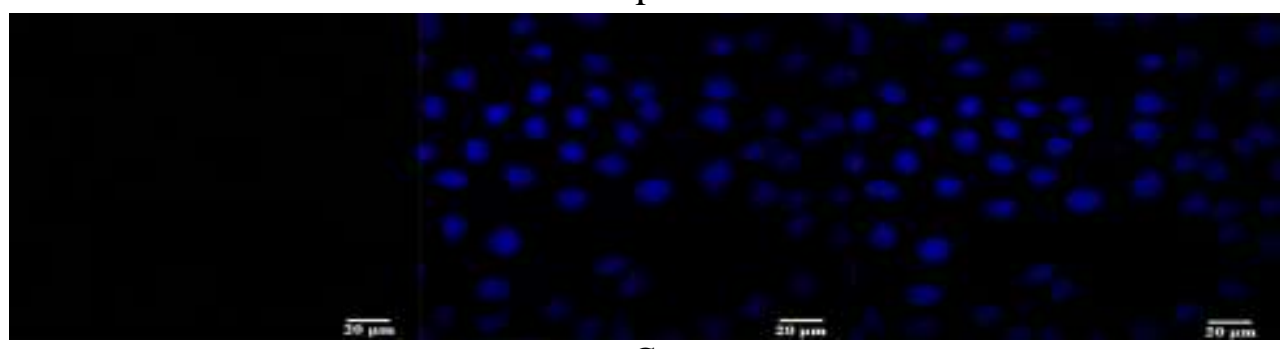

G

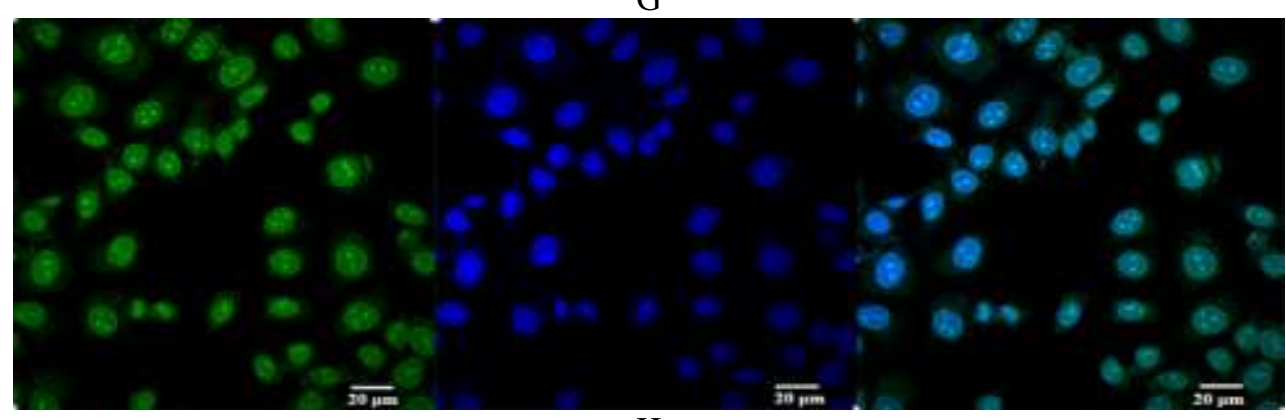

$\mathrm{H}$

Figure 5. Changes of SAM and SAH levels in L02 and HepG2 cells with Met stimulation as shown in IF cell smear.Three images show the same view, pictures in the left: FITC view; middle: DAPI view; right: FITC+DAPI.A: Normal liver cells L02 stained with anti-SAM antibody. Green fluorescein FITC indicates where SAM is. Blue fluorescein DAPI stains for nuclei. B: L02 cells were treated with $0.5 \mathrm{mM}$ Met for 24h, and then stained with the anti-SAM antibody 118-6. Met stimulates MATA1a expression in L02 and therefore SAM stained could be found in more areas including nuclei compared to (A). C: Hepatocellular carcinoma cells HepG2 stained with 118-6. D: HepG2 cells were treated with 0.5mM Met for 24h, and then stained with 118-6. Met inhibits MATA2a/2b expression in HepG2 and SAM stained weaker than (C).E, F, G, H: the same as in A, B, C, D above except that FITC signals indicated the amount and location of SAH in cells. 


\section{Discussion and Conclusions}

It is reported that in cultured rat hepatocytes MAT1aexpressionprogressively decreased and MAT2aexpression was induced.The switch in gene expression can beprevented by adding SAM.In cultured hepatocytes with decreasedMAT1aexpression SAM addition increased MAT1atranscription in a dose-dependentfashion. These findings identify SAM as akey molecule that differentially regulates MAT1aandMAT2aexpression and helps to maintain the differentiatedstatus of the hepatocyte.MATla mRNA and protein levels were absent in homozygous knockout mice.At 3 months, plasma methionine level increased $776 \%$ in knockouts. Hepatic SAM and glutathione levels were reduced by 74 and $40 \%$, respectively, whereas $S A H$, MTA, and global DNA methylation were unchanged.The expression of many inflammatory markers, including orosomucoid, amyloid, metallothionein, Fas antigen, and growth-related genes, including early growth response 1 and proliferating cell nuclear antigen, is increased in the knockout animal. The knockout mice are more susceptible to choline-deficient diet-induced fatty liver and would develop spontaneous macrovesicular steatosis and predominantly periportal mononuclear cell infiltration [11]. This implies that SAM is important in anti-inflammation and anti-proliferation of hepatocytes. SAM is able to differentially regulateMATlaandMAT2aexpression and helps to maintain the differentiatedstatus of the hepatocyte [12].

In observation of Met's regulation of MAT activity, two cell lines L02 and HepG2 are good models to study the two types of MAT. We employed the novel immunoassays to specifically and sensitively measure SAM level and MAT activity after incubation of cells with Met and GSNO. The investigation explains and links damages from free radicals to Met cycles, especially levels of SAM. As reported previously reduced level of SAM is related to inflammation and addition of SAM exhibited anti-inflammation effects [13], SAM may be mobilized and enriched when cells need to respond to certain emergency as seen with lung squamous cell carcinoma, T2N0M0, stage I (Figure 3D2).The reason should be related to increased SAM synthesis through enhanced MAT activity. Elevated SAM level promotes its aminopropylation pathway producing anti-inflammatory MTA and DNA-stabilizing and cell survival factors spermidine and spermine.The possibility of defective methylationleading to accumulation of SAMmay not be excluded too. Further study is needed to clarify this. GSNOis a free readical(NO donor). Inactivation of MAT-I/III activity, by incorporating an NO to the cysteine121 of MAT[14,15], might be the critical signal in response to NO-caused cell injuries. As a result, synthesis of SAM is impacted. The insufficient level of SAM leads to sacrifice of liver, causing liver proliferation andregeneration.In general liver damage causes increased free radicals, which in turn inhibits MAT-I/III activity. Inhibition of MAT activity prevents normal consumption and use of energy molecule ATP which endangers survival of liver cells. On the other hand, liver functions, regeneration differentiation and sensitivity to injury are impacted due to the reduced or defective SAM synthesis. SAM depleted animals show tissue injuries, necrosis and inflammatory infiltration. Given the critical roles of SAM and MAT in liver proliferation, differentiation and apoptosis, epigenetic regulation of MAT can be used as hepatocellular carcinoma therapy [16].

$0.5 \mathrm{mM}$ Met stimulated while GSNO inhibited MAT-I/III activities and methylation index increased afterMet stimulation of L02. 0.5mM Met inhibited while GSNO had no effects on MAT-II activities and methylation index decreased afterMet stimulation of HepG2. HepG2 cells exhibited deficient methylation andMet stimulation turned on DNA methyltransferase activity causing reduced SAM and increase SAH in nuclei. With specific anti-SAM, anti-SAH antibodies and immunoassays, cellular localization 
and quantification of SAM, SAH and MAT activity become possible, and the methods are quick, easy, sensitive and accurate. Methylation index can also be evaluated quickly and accurately to reflect methylation capability and status of cells. The novel methods used here will help address important questions related to methylation and Met cycle.

\section{References}

[1] J. D. Finkelstein. Methionine metabolism in mammals.J.Nutr. Biochem.1 (1990)228-236.

[2] J. M. Mato, L. Alvarez, P. Ortiz, M. A. Pajares. S-Adenosylmethionine synthesis: molecular mechanisms and clinical implications. Pharmacol.Ther.73 (1997)265-280.

[3] B. Gil, M. Casado, M.A. Pajares, L. Boscá, J. M. Mato, P. Martín-Sanz, L. Alvarez.

Differential expression pattern of S-adenosylmethioninesynthetaseisoenzymes during rat liver development.Hepatology. 24(1996) 876-881.

[4] S. C. Lu. S-Adenosylmethionine.Int J Biochem Cell Biol. 32(2000) 391-395.

[5] M. A. Avila, J. Mingorance, M. L. Martínez-Chantar, M. Casado, P. Martin-Sanz, L. Boscá, J. M. Mato. Regulation of rat liver S-adenosylmethioninesynthetase during septic shock: role of nitric oxide. Hepatology. 25(1997):391-396.

[6] A. B. Halim, L. LeGros, A. Geller, M. Kotb. Expression and functional interaction of the catalytic and regulatory subunits of human methionine adenosyltransferase in mammalian cells. J Biol Chem. 274(1999) 29720-29725.

[7] M. M. delPino, F. J. Corrales, J. M. Mato. Hysteretic behavior of methionine adenosyltransferase III. Methionine switches between two conformations of the enzyme with different specific activity.JBiol Chem. 275(2000) 23476-82342.

[8] M.L. Martinez-Chantar, M.U. Latasa, M. Varela-Rey, et al.L-Methionine Availability Regulates Expression of the Methionine Adenosyltransferase 2A Gene in Human Hepatocarcinoma Cells. J Biochem. 278(2003) 19885-19890.

[9] M.A. Avila, M.V. Carretero, E.N. Rodriguez, J.M. Mato.Regulation by hypoxia of methionine adenosyltransferase activity and gene expression in rat hepatocytes.Gastroenterology. 114(1998) 364-371.

[10] J. Fernández-Irigoyen, E. Santamaría, Y. H. Chien, W. Hwu,S. H. Korman, H. Faghfoury, A. Schulze, G. E. Hoganson, et al. Enzymatic activity of methionine adenosyltransferase variants identified in patients with persistent hypermethioninemia. Molecular Genetics and Metabolism. 101(2010) 172-177.

[11] S.C. Lu, L. Alvarez, Z. Huang, L. Chen,W. An, F. J. Corrales, M. A. Avila, G. Kanel, J.M. Mato. Methionine adenosyltransferase 1A knockout mice are predisposed to liver injury and exhibit increased expression of genes involved in proliferation. Biochemistry. 98(2001) 5560-5565.[12] E.R.Garci'a-Trevijano, M. U. Latasa, M.V.Carretero, C. Berasain, J. M. Mato, M. A. Avila. S-Adenosylmethionine regulates MAT1A and MAT2A gene expression in cultured rat hepatocytes: a new role for S-adenosylmethionine in the maintenance of the differentiated status of the liver. FASEB J. 14(2000) 2511-2518. 
[13] W. I. Najm, S. Reinsch, F. Hoehler, J. S. Tobis, P.W. Harvey. S-Adenosyl methionine (SAMe) versus celecoxib for the treatment of osteoarthritis symptoms: A double-blind cross-over trial. BMC Musculoskelet Disord. 5(20) 6-20.

[14] I. Pe'rez-Mato, C. Castro, F.A. Ruiz, F. J. Corrales, and J. M. Mato. Methionine adenosyltransferase S-nitrosylation is regulated by the basic and acidic amino acids surrounding the target thiol. J. Biol. Chem. 274(1999) 17075-17080.

[15] F. Ruiz, F. J. Corrales, C. Miqueo, and J. M. Mato. Nitric oxide inactivates rat hepatic methionine adenosyltransferase In vivo by S-nitrosylation. Hepatology.28(1998) 1051-1057.

[16] H. Yang, M.E. Cho, T. W. Li, H. Peng, K. S. Ko,J. M. Mato,et al. Epigenetic Regulation of Methionine Adenosyltransferase 1A: A Role for MicroRNA-Based Treatment in Liver Cancer? HEPATOLOGY. 57(2013) 2081 - 2084. 